\title{
Abordagem com foco ciência, tecnologia e sociedade no ensino de física: um estudo sobre a interferência de ondas entre $4 \mathrm{~g}$ e tv digital
}

\author{
Tamara Dias de Carvalho \\ IFRJ, Campus Arraial do Cabo \\ (tamaradc_@hotmail.com) \\ Cassiana Barreto Hygino \\ (cassiana.machado@ifrj.edu.br) \\ IFRJ, Campus Arraial do Cabo \\ Eros Izidoro Amaral \\ (eros.amaral@ifrs.edu.br) \\ IFRJ, Campus Arraial do Cabo
}

\begin{abstract}
Resumo: Nesta pesquisa, é apresentada a implementação de uma sequência de ensino com a abordagem Ciência, Tecnologia e Sociedade (CTS), por meio do método de Estudo de Caso, em uma turma do ensino médio integrado ao curso técnico em Informática, do Instituto Federal de Educação, Ciência e Tecnologia do Rio de Janeiro (IFRJ). A sequência de ensino objetivou a aprendizagem do conteúdo de interferência de ondas de forma contextualizada, por meio do tema: a interferência entre 4G e TV digital. Durante o desenvolvimento, os alunos realizaram pesquisas sobre a polêmica da mudança de faixas de frequência ocorrida no Brasil e apresentaram experimentos sobre interferência, que ajudaram a pensar em soluções para o Estudo de Caso proposto. Como resultado, os estudantes adquiriram conhecimentos sobre o fenômeno da interferência e também sobre a organização e política de meios de comunicação que utilizam ondas eletromagnéticas, no caso, a internet móvel e o sinal de TV, desenvolvendo, ao final, uma posição crítica e responsável em relação ao assunto.
\end{abstract}

Palavras-chave: Ensino de Física; CTS; Ondas Eletromagnéticas.

\section{Science, technology and society approach in physics teaching: a study about wave interference between $\mathbf{4 g}$ and digital tv}

\begin{abstract}
In this research, an implementation of a teaching sequence was implemented with the Science, Technolgy and Society (STS) approach and the Case Study Method in a High School class of the technical course in computer science from Instituto Federal de Educação, Ciência e Tecnologia do Rio de Janeiro (IFRJ). The teaching sequence aimed at learning the content of wave interference in a contextualized way, through the theme: the interference between $4 \mathrm{G}$ and digital TV. Through the development of this activity the students have done researches about the controversy wave frequency change in Brazil and presented experiments about interference that helped them to think in a denouement for the proposed Case Study. As result, the students gained
\end{abstract}


knowledge about the interference phenomenon, the media organization and policy that use electromagnetic waves, as mobile phone internet and TV signal, developing at the end a critical and responsible position on the subject.

Keywords: Physics Teaching; STS; Electromagnetic Waves.

\section{INTRODUÇÃO}

$\mathrm{Na}$ vida atual, utilizam-se constantemente aparelhos tecnológicos com o uso da radiação. Muitas pessoas têm um celular, um computador ou um forno de micro-ondas em casa. É de grande importância que as pessoas entendam como tais aparelhos funcionam, os riscos e benefícios de determinada radiação para usá-los de forma responsável e consciente, entendendo recursos de diagnóstico médico (tomografias e radiografias) ou que tipo de material não se deve colocar em um microondas.

Nesse sentido, o Ensino de Física precisa ultrapassar algo meramente tradicional, que se traduz em fórmulas e memorização, para um ensino em que o aluno é o protagonista e seja capaz de relacionar a física com fenômenos, tecnologias e problemas sociais a sua volta. A utilização da abordagem Ciência, Tecnologia e Sociedade (CTS) atende este anseio, pois relaciona conceitos científicos a questões sociais. Os estudantes são levados a compreender os fenômenos técnicos e científicos que estão à sua volta contribuindo para a formação cidadã na escola. Para isso, o aluno adquire conhecimentos, habilidades e valores necessários. Dentre os conhecimentos e habilidades estão: o pensamento lógico, a escrita e a oralidade, o exercício da cidadania e a autoestima (SANTOS e MORTIMER, 2002, p. 23).

Para alcançar tais objetivos, é necessária a utilização de metodologias de ensino que coloquem o aluno com um papel privilegiado no processo de ensinoaprendizagem. Desse modo, pergunta-se: como tratar conteúdos de física por meio de um tema social relevante, de modo a contribuir com a aprendizagem de conteúdos e com a formação cidadã dos estudantes? 
A fim de responder essa questão, foi elaborada e aplicada uma sequência de ensino, com o objetivo de trabalhar o conteúdo de interferência de ondas de forma contextualizada. Para isso, foi utilizada como metodologia de ensino, a abordagem CTS e o método de Estudo de Caso. Essa metodologia traz o Estudo de um Caso para ser investigado pelos alunos. Nessa sequência de ensino, o Caso tem como tema a interferência de ondas entre $4 \mathrm{G}$ e TV digital. Durante o desenvolvimento da metodologia, os estudantes compreendem o conteúdo científico e também realizam pesquisas acerca do tema, a fim de proporem soluções para o Caso tratado.

\section{A ABORDAGEM CIÊNCIA, TECNOLOGIA E SOCIEDADE}

Desde a década de 60 , do século XX, currículos com ênfase em CTS vêm sendo desenvolvidos. De acordo com Pinheiro et al. (2009):

Ciência, Tecnologia e Sociedade - CTS corresponde ao estudo das inter-relações existentes entre a ciência, a tecnologia e a sociedade, constituindo um campo de trabalho que se volta tanto para a investigação acadêmica como para as políticas públicas. Baseia-se em novas correntes de investigação em filosofia e sociologia da ciência, podendo aparecer como forma de reivindicação da população para atingir uma participação mais democrática nas decisões que envolvem o contexto científicotecnológico ao qual pertence. Para tanto, o enfoque CTS busca entender os aspectos sociais do desenvolvimento tecnocientífico, tanto nos benefícios que esse desenvolvimento possa estar trazendo, como também as consequências sociais e ambientais que poderá causar. (PINHEIRO et al., 2009, p.2-3).

A escola passou a ser criticada por não estar contribuindo com uma formação para a ciência e a tecnologia que cultive a atitude crítica e participativa com respeito a esses campos de saberes. Tornou-se, então, imprescindível a aquisição de conhecimentos científicos e tecnológicos, de modo que a população pudesse, além de ter acesso às informações sobre o desenvolvimento nessa área, ter também condições de avaliar e participar das decisões que venham a atingir o meio onde vive. A esse respeito, Bazzo (1998) comenta: 
O cidadão merece aprender a ler e entender muito mais do que conceitos estanques - a ciência e a tecnologia, com suas implicações e consequências, para poder ser elemento participante nas decisões de ordem política e social que influenciarão o seu futuro e o dos seus filhos. Bazzo (1998, p. 34).

Desse modo, os currículos escolares e a formação de professores precisam ser repensados, uma vez que as aulas de ciências devem envolver questões que emergem da vida cotidiana oriundas do desenvolvimento científico e tecnológico.

O objetivo central da abordagem CTS no ensino médio é o de desenvolver a alfabetização científica e tecnológica dos cidadãos, auxiliando o aluno a construir conhecimentos, habilidades e valores necessários para tomar decisões responsáveis sobre questões de ciência e tecnologia na sociedade e atuar na solução de tais questões (AIKENHEAD, 1994).

Pinheiro, Silveira e Bazzo (2009) ressaltam que:

O enfoque CTS que venha a ser inserido nos currículos é apenas um despertar inicial no aluno, com o intuito de que ele possa vir a assumir essa postura questionadora e crítica num futuro próximo. Isso implica dizer que a aplicação da postura CTS ocorre não somente dentro da escola, mas, também, extramuros (Pinheiro et al, 2009, p.77).

Acredita-se, assim, que a introdução do enfoque CTS, no ensino médio, poderia promover um ensino-aprendizagem que propiciasse ao aluno habilidade de discutir sobre assuntos relacionados com a ciência, a tecnologia e a sua implicação social nos aspectos ligados à sua área de atuação, o que poderia leválo, enfim, a uma autonomia profissional crítica.

\section{DESENVOLVIMENTO DA PESQUISA}

A proposta didática foi aplicada no Instituto Federal de Educação, Ciência e Tecnologia do Rio de Janeiro - Campus Arraial do Cabo, em uma turma do curso técnico em Informática Integrado ao Ensino Médio. O curso é voltado para aqueles que desejam cursar o ensino médio com uma educação profissional, recebendo, 
ao final do curso, o certificado de conclusão do Ensino Médio e o diploma de técnico.

O curso tem duração de três anos e seis meses, sendo dividido em sete semestres. A disciplina de física é ministrada do primeiro ao quinto semestre, tendo quatro aulas de 45 minutos por semana nos quatro primeiros períodos, e duas aulas de 45 minutos por semana no quinto período. A aplicação da proposta ocorreu no segundo bimestre do quinto período da turma, na qual havia 18 alunos.

Para o desenvolvimento da proposta, foi criada uma sequência de ensino, a qual utiliza a abordagem CTS e, como metodologia de ensino, o método do Estudo de Caso. Nesse método, é criado um Caso, o qual se baseia em narrações sobre pessoas que enfrentam dilemas e têm de tomar alguma decisão. O Caso é apresentado ao aluno, quando o aluno é levado a ler, compreender o contexto do problema e a se acostumar com os personagens, para posteriormente pensar em uma solução para o problema e saber argumentar a favor da solução encontrada por ele, que não necessariamente deve ser a única (SÁ; FRANCISCO; QUEIROZ, 2007). Esse método é baseado no Problem Based Learning (Aprendizado Baseado em Problemas - PBL), que se originou na Escola de Medicina da Universidade de McMaster, no Canadá. Ele foi criado com o intuito de levar os alunos a enfrentar problemas reais referentes à sua área de estudo, incentivando o pensamento crítico e a tomada de decisões. De tal maneira, o aluno passa a ser atuante no seu aprendizado, buscando seu próprio conhecimento (SÁ; FRANCISCO; QUEIROZ, 2007).

O Estudo de Caso é trabalhado em três passos, assim como sugerem Linhares e Reis (2008 apud Rangel e Guimarães, 2014): no primeiro passo, os professores devem expor o tema para os alunos, que depois se dividem em pequenos grupos ou trabalham individualmente para ler o Caso e começam a busca pela resolução da questão. Essas primeiras soluções dos estudantes para o Caso se baseiam nas suas ideias prévias, ou seja, em conhecimentos que já possuem acerca do tema de estudo.

No segundo passo, ocorre uma discussão em que o professor apresenta os pontos importantes do caso, além de realizar aulas expositivas sobre o conteúdo 
de estudo, experimentos, pesquisas sobre o tema proposto, entre outras atividades.

No terceiro passo, os estudantes devem responder novamente o Caso, incorporando os conhecimentos aprendidos.

Para o desenvolvimento da sequência de ensino, construiu-se o Caso "Ou Tv ou Celular", mostrado no Quadro 1:

Quadro 1: Estudo de Caso: Ou TV ou Celular.

\section{Ou TV ou Celular}

Pedro, de 17 anos e morador da cidade de Cabo Frio, estava muito ansioso para ver o show da sua banda favorita, Metallica, no Rock in Rio. Infelizmente, não tinha conseguido comprar o ingresso do dia a tempo, então iria assistir pela sua nova TV. Enquanto via o show, decidiu escrever um tweet:

"Vendo show do Metallica. Mt bom!"

O tweet não foi publicado e a imagem da sua TV travou por um momento.

- O que aconteceu? Mãeeee, a TV nova está com problema, acabou de travar a imagem e o som ficou com um zumbido. - disse Pedro.

- Ah filho, isso aconteceu comigo de tarde também, você está usando o (4G) no celular? - respondeu sua mãe, que estava na cozinha lavando a louça.

- Estou sim, por quê?

- Então é isso, a vizinha disse que acontece o mesmo com ela, sempre que usa a internet no celular juntos acontecem essas coisas.

- Caramba, vou sempre ter que escolher um deles?!

Se você fosse um amigo de Pedro, como explicaria o motivo pelo qual a TV e o celular estão com problemas de funcionamento ao mesmo tempo?

Fonte: Autores. 
O tema "A Interferência entre 4G e TV Digital" foi escolhido porque é atual e relacionado ao curso que os alunos da escola estão realizando. Atualmente, no Brasil, o serviço de banda larga móvel de quarta geração (4G) utiliza a faixa de frequência de 2,5 gigahertz, mas agora a faixa de 700 megahertz (frequências de $698 \mathrm{MHz}$ a $806 \mathrm{MHz}$ ) será leiloada para o uso do 4G, podendo render $\mathrm{R} \$ 8$ bilhões para o governo. Essa faixa permite maior alcance e apresenta menos interferências por possuir uma frequência menor.

O leilão da faixa dos 700 megahertz vem causando polêmicas no Brasil por causa da possível interferência com a TV digital, que opera em uma faixa de frequência muito próxima, tornando provável que um serviço invada a faixa do outro. Essa interferência poderia prejudicar tanto o serviço do $4 G$ quanto o da TV digital. A situação causou um conflito entre o setor de radiodifusão e as operadoras de telefonia, podendo adiar o leilão da faixa.

A Sociedade Brasileira de Engenharia de Televisão (SET) contratou a Universidade Mackenzie para realizar testes sobre a interferência do 4G com a TV digital. Os testes, feitos em gaiolas blindadas eletromagneticamente, mostraram como resultado a possível interferência. Se estiverem perto de uma estação-base, de antenas de celular e antenas que se encontram nas casas, é possível ocorrer saturação ou interferência. A Agência Nacional de Telecomunicações (Anatel) elaborou um regulamento para que não ocorra interferência entre esses serviços, no qual inclui aplicação de filtros e alteração de potências de transmissão. Mesmo com essas regras, a SET diz que as medidas estabelecidas pela Anatel são insuficientes.

A faixa dos 700 megahertz é usada para o 4G em outros países e funciona sem ocasionar interferências. A diferença é que, em países como os Estados Unidos, as faixas de frequência são bem definidas, com um espaço mínimo entre uma e outra. Além disso, as determinações são respeitadas pelas emissoras de TV e telefonia móvel e também rigidamente fiscalizadas, com instrumentos de medição de qualidade.

$\mathrm{Na}$ sequência de ensino, no primeiro passo, de acordo com a estrutura que sugere Linhares e Reis (2008 apud Rangel e Guimarães, 2014), foi apresentado o 
Estudo de Caso "TV ou Celular" aos alunos, e esses tiveram que respondê-lo com seus conhecimentos prévios.

No segundo passo, foram ministradas aulas sobre Ondulatória. Nessas aulas, os alunos tomaram conhecimento do comportamento de uma onda eletromagnética e de seus fenômenos, como a interferência que ocorre no Caso em questão. Os alunos também foram divididos em dois grupos, cada qual com nove alunos, para apresentar um trabalho e um experimento. Um grupo tinha de explicar o que é 4G, TV Digital e como ocorre a interferência. Seu experimento era uma interferência de rádio, para o qual utilizaram uma fonte de energia com fios de cobre em uma lima de ferro e um rádio, de acordo com a Figura 1. Ao arrastar um fio de cobre na lima de ferro, ocorria uma interferência no sinal do rádio.

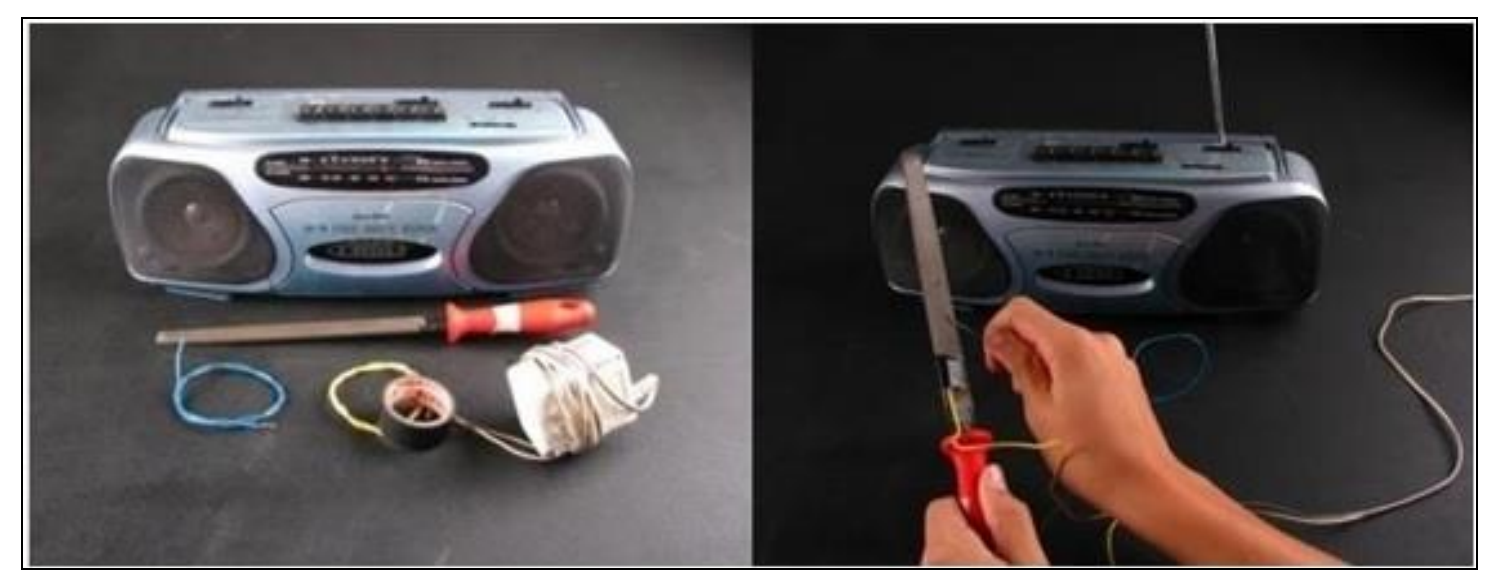

Figura 1 - Rádio, lima de ferro e fios condutores. Fonte: www.pontociencia.org.br (2011)

O outro grupo apresentou as questões socioeconômicas que envolviam este Caso, explicando o motivo da pressa em colocar o $4 \mathrm{G}$ na banda de $700 \mathrm{MHz}$, no Brasil, e também porque não ocorria interferência em outros países que já tinham essa faixa de frequência para o 4G. Como experimento, utilizaram uma lanterna e uma câmera de celular para mostrar interferências construtivas e destrutivas. Ao incidir a luz da lanterna no orifício da câmera, foi possível observar na tela faixas brancas e pretas resultantes da interferência ocasionada pela luz, Figura 2. 


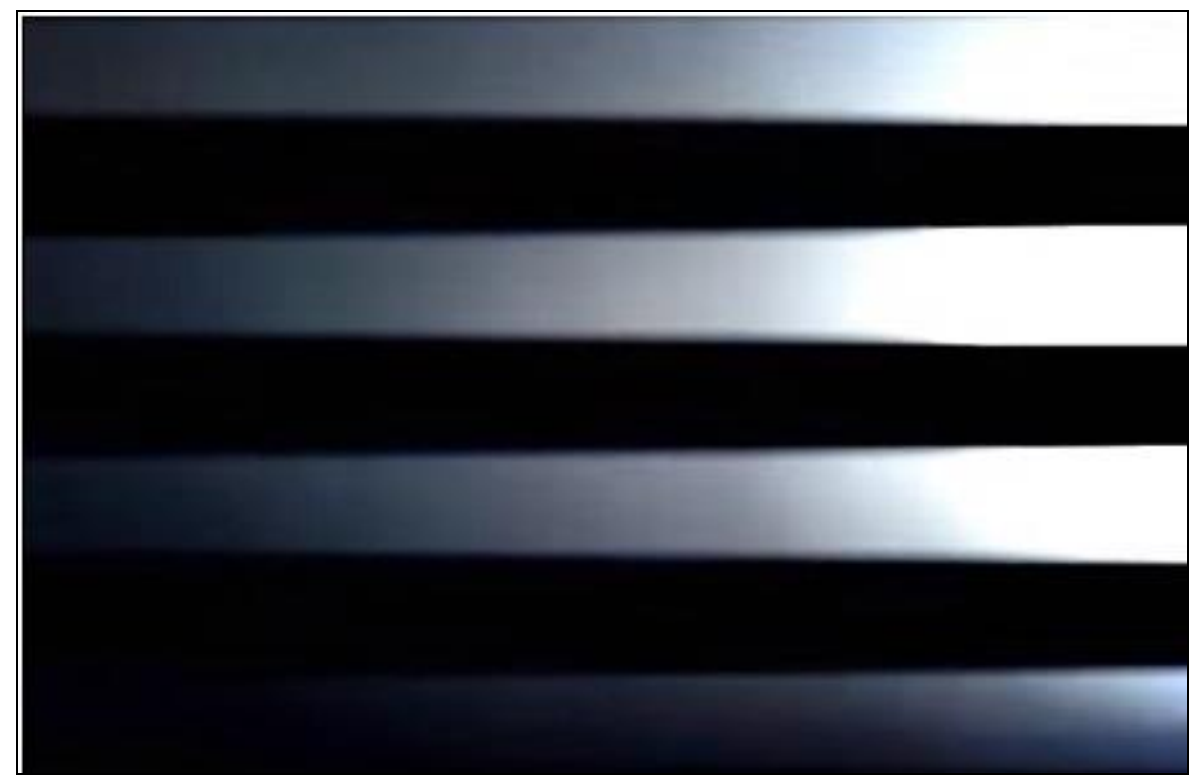

Figura 2 - Faixas brancas e pretas.

Fonte: www.youtube.com/watch?v=cJMNjC2Z7Ls.

No terceiro passo, os alunos tiveram de responder novamente ao Estudo de Caso, considerando os novos conhecimentos sobre o assunto.

\section{METODOLOGIA}

A pesquisa realizada é de caráter qualitativo, ou seja, ao invés dos dados serem quantificados para se ter um resultado preciso, foram analisados os assuntos e o conhecimento demonstrado em virtude das respostas dos alunos. A pesquisa qualitativa tem como objetivo melhorar a compreensão dos fenômenos que estão sendo examinados, de forma sistemática e criteriosa, de acordo com Moraes e Galiazzi (2007 apud HYGINO et al., 2013).

Utilizamos a Análise Textual Discursiva - ATD para podermos analisar as respostas ao Estudo de Caso nos passos um e três da Sequência Didática. A pesquisa em questão não tem como objetivo testar hipóteses, ela visa somente a compreensão (MORAES E GALIAZZI, 2007, apud HYGINO et al., 2013). Os textos analisados são chamados de corpus, que no caso desta pesquisa são as respostas dos alunos. 
A ATD é composta por três etapas, que fazem parte de um processo cíclico:

a) Desmontagem dos textos ou unitarização: nessa etapa, os textos são minuciosamente analisados, para serem fragmentados em unidades de sentido de acordo com os fenômenos estudados, tendo sempre cuidado para se manter o contexto de onde foi retirado o fragmento. É importante dar atenção aos detalhes nessa etapa, e é o pesquisador que decide o quanto o texto deverá ser fragmentado.

b) Estabelecimento de relações ou categorização: nessa etapa serão criadas relações entre as unidades, tanto as empíricas quanto as teóricas. As unidades são comparadas e categorizadas de acordo com suas semelhanças. Podemos afirmar que a categorização é um processo de criação, ordenamento, organização e síntese (MORAES; GALIAZZI, 2007, apud HYGINO et al., 2013, p. 78).

c) Comunicação ou produção de metatextos: o propósito agora é elaborar um texto descritivo e interpretativo a partir das categorias, que são os metatextos. Nessa etapa, é possível ter uma compreensão do todo, devido à profunda realização das outras etapas.

\section{RESULTADOS}

Para preservar a identidade dos alunos eles foram chamados de: AN, CA, FE, FA, GM, GL, IC, JP, JV, KA, LO, LF, MV, MF, PA, RO, TA, YG. A partir da ATD, dividiram-se as respostas dos alunos aos passos inicial e final, em dois metatextos: Relação da Física com o Funcionamento dos Aparelhos e Aspectos Sociais e Econômicos.

\subsection{Relação da Física com o funcionamento dos aparelhos}

Referente aos aspectos físicos na transmissão de informação da TV e do celular, no primeiro passo, os alunos conseguiram identificar que o problema que ocorria no Estudo de Caso era uma interferência, como por exemplo, o estudante 
AN: "As ondas se encontram fazendo interferência em ambos os aparelhos" (AN). Apesar de terem identificado o problema físico, não apresentaram conceitos completamente corretos quanto ao assunto.

Os alunos GL, KA e LO acreditavam que a transmissão da TV e do Celular tivessem diferenças em suas características. "Por, os sinais, serem diferentes, da TV e do 4G, um interfere no outro, assim, perdendo a transmissão dos dois" (LO). $O$ aluno $G L$ disse que a onda da TV e do $4 G$ tem tamanhos diferentes, enquanto 0 aluno KA acreditava que as frequências eram diferentes, e, por isso, houve interferência.

"A interferência é o fenômeno resultante da superposição de duas ou mais ondas" (RAMALHO; FERRARO; TOLEDO, 2007, p. 508). Essa superposição, ou interferência, acontece quando duas ondas têm a mesma frequência.

De acordo com Barreto e Xavier (2013, p.318): "Os tipos de onda eletromagnética (intervalos do espectro eletromagnético) são de mesma natureza e possuem mesma velocidade de propagação, apresentando frequência, comprimento de onda e energia diferentes", e como as ondas da TV e do Celular são da mesma faixa, pela equação fundamental ondulatória $(v=\lambda f)$, concluímos que o comprimento de onda é o mesmo quando ocorre uma interferência.

O estudante LF citou o espectro eletromagnético, demonstrando conhecimento sobre a matéria: "Eu diria que é uma interferência do sinal que é fornecido dos aparelhos em suas respectivas tecnologias que por acaso estão na mesma frequência no espectro eletromagnético" (LF). O aluno PA falou sobre o comprimento de onda: "O comprimento de ondas deve ter interagido, o que gera uma alteração" (PA).

Ao ocorrer uma interferência, os vales ou cristas das ondas se sobrepõem, podendo ser uma interferência destrutiva ou construtiva. Chamamos os pontos mais altos da onda de crista, e os mais baixos de vales ou depressões (BARRETO; XAVIER, 2013). Elas dependem da amplitude da onda, que pode ser maior ou menor de acordo com a energia transportada.

Já no terceiro passo, os alunos apresentaram uma melhor compreensão quanto à interferência. Os alunos CA e TA comentaram a faixa de frequência 
utilizada pelos aparelhos: "Pois a TV digital funciona com uma frequência/faixa muito próxima à da internet $4 \mathrm{G}$, e assim os sistemas se anulam, causando a interferência e ruídos na TV" (CA).

Os alunos YG e FE comentaram que a interferência ocorrida no caso era destrutiva: "Eu explicaria para Pedro que o $4 G$ e a TV funcionam em frequências próximas, isso faz com que ocorra uma interferência destrutiva, que impede o envio do tweet e causa o travamento da imagem" (FE).

O estudante YG comentou sobre a fase das ondas: "A onda do $4 G$ e da TV são similares com fases opostas, sendo de fases opostas, o resultado é uma interferência destrutiva onde as amplitudes se cancelam, porém, a velocidade é mantida" (YG).

Assim como os alunos afirmaram, duas fontes com mesma frequência e gerando pulsos com concordância de fases terão uma interferência destrutiva no ponto em que elas se encontram em oposição de fase (BARRETO; XAVIER, 2013, p. 320).

\subsection{Aspectos sociais e econômicos}

No primeiro passo, os alunos não conseguiram relacionar nenhum aspecto político à interferência ocorrida nas ondas da TV e do Celular. Isso demonstra que a mudança da faixa de frequência utilizada pelo $4 G$ não é conhecida pelos alunos, isso pode ser porque o assunto não é divulgado o bastante.

No terceiro passo, os alunos KA e MV comentaram sobre as faixas de frequências dos aparelhos utilizadas no Brasil: "Porque a TV digital e o 4G, aqui, no Brasil, estão funcionando em frequências muito próximas, na faixa de $700 \mathrm{MHz}$, por isso quando utilizadas simultaneamente, as ondas se sobrepõem causando interferências" (KA).

A operadora TIM já começou a utilizar a faixa de $700 \mathrm{MHz}$ para o 4G, em 2016 (MINHA OPERADORA, 2016). Antes, essa faixa era utilizada para a transmissão do sinal analógico de TV, que tem previsão de acabar em todo o Brasil em 2019. Em 2014, a Anatel admitiu que existem conflitos de sinais entre a TV Digital e a 
tecnologia 4G, problemas já apontados antes pela Sociedade Brasileira de Engenharia de Televisão (SET) (CANAL TECH, 2014).

O aluno TA apresentou, em sua resposta, como a questão das faixas de frequência é resolvida nos EUA, comparando também o investimento dos países nessa questão: em países como os EUA, os equipamentos que verificam as frequências são precisos e a fiscalização das operadoras eficazes, mas, no Brasil, infelizmente, isso não ocorre (TA). Além disso, comentou também sobre o custo com antenas para as empresas com a faixa de $700 \mathrm{MHz}$ : porque como é uma frequência menor, consegue maior alcance em ambientes fechados e assim as operadoras utilizam menos antenas (TA).

O site Olhar Digital fez uma entrevista com Alessandro Cunha, diretor de tecnologia da Tech Training, e nela, ele declara que não há problemas com o uso dessa faixa para o $4 G$ porque as frequências são bem definidas, além de serem constantemente fiscalizadas com aparelhos precisos pelas agências responsáveis (OLHAR DIGITAL, 2014).

O uso de frequências mais altas como a de $2600 \mathrm{MHz}$, que é ainda utilizada no $4 \mathrm{G}$, tem um alcance menor em relação a frequências mais baixas. Para as operadoras poderem cobrir mais cidades com essa rede móvel, elas teriam que pôr mais antenas utilizando a faixa de $2600 \mathrm{MHz}$ do que a de $700 \mathrm{MHz}$, o que traz um custo maior (TECHNOBLOG, 2014).

\section{CONSIDERAÇÕES FINAIS}

No trabalho apresentado, elaborou-se uma sequência de ensino com abordagem CTS, tendo como metodologia de ensino o método de Estudo de Caso. O Caso trabalhado teve como tema: "A Interferência de ondas entre 4G e TV Digital" e relaciona o conteúdo de ondas eletromagnéticas com a mudança da utilização da faixa de $700 \mathrm{MHz}$, no Brasil. A sequência de ensino foi implementada em uma turma de ensino médio integrado ao técnico de informática, curso composto por sete semestres, em uma turma do quinto semestre. Em todos os 
três passos do desenvolvimento da pesquisa, os alunos foram convidados a analisar os dados científicos e pensar de forma crítica com relação às mudanças políticas que ocorrem no país.

As respostas dos alunos, aos passos iniciais e finais do Estudo de Caso, foram analisadas mediante a ATD. Nas respostas, foi possível perceber que os estudantes compreenderam o conceito de interferência, verificando que este fenômeno acontece em ondas eletromagnéticas quando há uma superposição as ondas. Essa superposição acontece quando as ondas têm a mesma frequência. No que se refere às relações sociais e econômicas, percebemos que os alunos passaram a ter conhecimento sobre a legislação que envolve a divisão das faixas de frequência de acordo com cada tecnologia e empresa. Além disso, puderam perceber questões políticas relacionadas à fiscalização de empresas e comparar como isso é realizado no Brasil e em outros países.

Ao analisar os dados coletados, pode-se perceber como uma abordagem CTS junto a um Estudo de Caso favorece que o aluno aprenda o conteúdo relacionando-o com questões socioeconômicas a sua volta. O uso da ciência não é neutro aos interesses e relações políticas existentes no mundo, por esse motivo, é importante que, em sala de aula, esses assuntos também sejam apresentados de forma conjunta, para que os alunos tenham atitudes críticas e responsáveis no mundo.

A pesquisa mostrou uma sequência de ensino com o enfoque CTS sobre o conteúdo de ondas, entretanto, esse tipo de abordagem pode ser usado em diversos assuntos, relacionados não só à Física como também a outras áreas ou ainda de forma interdisciplinar. Dessa maneira, esperam-se aplicar, futuramente, novos Estudos de Caso aos alunos, relacionando diferentes assuntos, de forma a ampliar seu leque de conhecimento e verificar como se dá resposta a essas novas questões trazidas por meio do mesmo tipo de abordagem. É possível ainda que se realizem estudos por meio de outras formas de abordagem, de modo a comparar o conhecimento adquirido pelos alunos por meio de diferentes maneiras. 


\section{REFERÊNCIAS}

AIKENHEAD, G. S. What is STS science teaching? SOLOMON, J.; AIKENHEAD, G.S. STS education: international perspectives on reform. New York: Teachers College Press, 1994. p.47-59

BARRETO, Benigno; XAVIER, Claudio. Física aula por aula 3. 2. ed. São Paulo: FTD, 2013. 320 p.

BAZZO, W. A. Ciência, Tecnologia e Sociedade: e o contexto da educação tecnológica. Florianópolis: Ed. da UFSC, 1998.

CANAL TECH, Anatel admite problemas de interferência entre TV digital e rede 4G. Disponível em: <https://canaltech.com.br/noticia/anatel/Anatel-admite-problemas-deinterferencia-entre-TV-digital-e-rede-4G/>. Acesso em 15 de outubro de 2016.

RANGEL, Fernanda Cristina da Silva; GUIMARÃES, Manoela Barros. Estudo de Casos: Um método didático para o Ensino de Oxirredução. 2014. 74f. Monografia (Licenciatura em Química) - Instituto Federal de Educação, Ciência e Tecnologia Fluminense, Campos dos Goytacazes.

MINHA OPERADORA, Primeira rede $4 \mathrm{G}$ na faixa de $700 \mathrm{MHz}$ do Brasil é da TIM. Disponível em: <http://www.minhaoperadora.com.br/2016/06/primeira-rede-4g-na-faixade-700-mhz-do-brasil-tim.html>. Acesso em 15 de outubro de 2016.

HYGINO, C.B. MARCELINO, V.S. LINHARES, M. P. Modelos didáticos presentes na formação de futuros professores de química e física da região norte do estado do Rio de Janeiro, Brasil: encontros e desencontros entre concepções e formação, REIEC Volumen8Nro .2, 2013.

OLHAR DIGITAL, Entenda o atrito entre 4G e TV digital no Brasil. Disponível em: $<$ http://olhardigital.uol.com.br/video/entenda-o-atrito-entre-4g-e-tv-digital-nobrasil/41782>. Acesso em 2 de novembro de 2015.

PINHEIRO, N. A. M.; SILVEIRA, R. M. C. F.; BAZZO , W. A. O contexto científicotecnológico e social acerca de uma abordagem crítico-reflexiva: perspectiva e enfoque. Revista Iberoamericana de Educação, Madrid, v. 49, n. 1, p. 1-14, mar. 2009.

RAMALHO, Francisco; FERRARO, Nicolau Gilberto; TOLEDO, Paulo Antônio de. Os Fundamentos da Física 3. 9ed. São Paulo: Moderna, 2007. 508 p.

RANGEL, Fernanda Cristina da Silva; GUIMARÃES, Manoela Barros. Estudo de Casos: Um método didático para o ensino de Oxirredução. 2014. 74 f. Monografia (Ciências da Natureza - Licenciatura em Química) - Instituto Federal de Educação, Ciência e Tecnologia Fluminense, Campos dos Goytacazes.

SÁ, L. P.; FRANCISCO, C. A.; QUEIROZ, S. L. Estudos de Casos em Química. Química Nova, v.30, n.3, p.731-739, 2007. 
SANTOS, Wildson; MORTIMER, Eduardo. Uma análise de pressupostos teóricos da abordagem C-T-S (Ciência - Tecnologia - Sociedade) no contexto da educação brasileira. Dezembro 2002, 23 p.

TECHNOBLOG, O que o $4 G$ de $700 \mathrm{MHz}$ muda na sua vida. Disponível em: <https://tecnoblog.net/166950/4g-700-mhz-brasil/>. Acesso em 15 de outubro de 2016. 